\title{
Experimental Characterization of a Composite Morphing Radiator Prototype in a Relevant Thermal Environment
}

\author{
Christopher L. Bertagne*, Jorge B. Chong ${ }^{\dagger}$, John D. Whitcomb ${ }^{\ddagger}$, Darren J. Hartl ${ }^{\S}$ \\ Texas A\&M University, College Station, Texas, 77843 \\ Lisa R. Erickson ${ }^{\mathbb{I}}$ \\ NASA Johnson Space Center, Houston, Texas, 77058
}

\begin{abstract}
For future long duration space missions, crewed vehicles will require advanced thermal control systems to maintain a desired internal environment temperature in spite of a large range of internal and external heat loads. Current radiators are only able to achieve turndown ratios (i.e. the ratio between the radiator's maximum and minimum heat rejection rates) of approximately 3:1. Upcoming missions will require radiators capable of 12:1 turndown ratios. A radiator with the ability to alter shape could significantly increase turndown capacity. Shape memory alloys (SMAs) offer promising qualities for this endeavor, namely their temperature-dependent phase change and capacity for work. In 2015, the first ever morphing radiator prototype was constructed in which SMA actuators passively altered the radiator shape in response to a thermal load. This work describes a follow-on endeavor to demonstrate a similar concept using highly thermally conductive composite materials. Numerous versions of this new concept were tested in a thermal vacuum environment and successfully demonstrated morphing behavior and variable heat rejection, achieving a turndown ratio of 4.84:1. A summary of these thermal experiments and their results are provided herein.
\end{abstract}

\section{Introduction}

For future crewed spacecraft targeting exploration beyond Low Earth Orbit (LEO) difficult thermal control requirements will need to be satisfied. The thermal control system (TCS) will be expected to maintain precise internal temperatures despite large large variations in the external thermal environment and internal system heat loads. In many cases the TCS will be required to reject a high heat load to a warm orbital environment and a low heat load to colder transit environments . ${ }^{1}$ This inverse relationship between the thermal environment and the heat rejection needs of the mission/spacecraft requires thermal control devices with a high turndown ratio. The turndown ratio, defined as the ratio between the maximum and minimum heat rejection capabilities of the TCS, is a primary measurement of TCS performance.$^{2}$ More precisely, this ratio involves the maximum heat rejection rate in the hottest environment and the miminimum heat rejection rate in the coldest environment. The turndown ratio of a TCS is therefore measured relative to the particular thermal environments in which it is designed to operate. Future missions beyond LEO are predicted to require turndown ratios between 6:1 and 12:1. ${ }^{3}$ The highest turndown ratio a state-of-the-art TCS achieves is 3:1 (in LEO).$^{4}$ Here, the TCS requires a heat transfer fluid with a low freezing point ${ }^{5}$ Such fluids, however, are typically toxic (e.g. ammonia on the International Space Station), which pose a hazard to the crew. This limits the TCS architecture to a two-loop (multi-fluid) design that isolates the crew from possible exposure to the harmful fluid.

Several efforts have been undertaken to improve TCS performance through the development of variable heat rejection radiators. ${ }^{5}$ Examples include roll-out fin radiators,${ }^{6}$ freezable radiators,${ }^{2}$ digital radiators,${ }^{7}$ and variableemissivity radiators.$^{8-11}$ This work supports the development of a novel type of radiator, known as a variable-geometry or morphing radiator, ${ }^{12}$ and provides details regarding the thermal characterization of various prototypes that were built.

\footnotetext{
*NASA Space Technology Research Fellow, Department of Aerospace Engineering, 3141 TAMU, AIAA Student Member

${ }^{\dagger}$ Undergraduate Researcher, Department of Aerospace Engineering, 3141 TAMU

¥Professor, Department of Aerospace Engineering, 3141 TAMU, AIAA Member.

$\S$ Assistant Professor, Department of Aerospace Engineering, 3141 TAMU, AIAA Member.

IThermal Engineer, Crew and Thermal Systems Division, 2101 NASA Parkway.
} 
Shape memory alloys are ideally suited for the design of morphing radiators. SMAs undergo changes in shape in response to changes in temperature ; a reversible process due to the stress- and temperature-dependent transformation between two solid material phases: austenite and martensite. ${ }^{13}$ Applied as mechanical actuators on a morphable radiator they would exploit energy transferred between the environment and working fluid to change the radiator shape with no need for external power, control, or sensing instrumentation. Such a radiator has the potential to achieve the high turndown ratios necessary to enable single-loop thermal control of a vehicle using a non-toxic, high-freezing-point working fluid, such as a propylene glycol/water solution (PGW). Trade studies have shown that a single-loop TCS with a morphing radiator system would reduce the TCS mass by approximately $25 \%$ compared with a standard two-loop design. ${ }^{3}$ Thus, the morphing SMA radiator concept has the potential to revolutionize current TCS technology by decreasing system mass and complexity, while increasing versatility.

\section{Description of Morphing Radiator Concept}

The primary goal of this work was to develop and conduct experimental studies on a structurally and thermally optimized composite morphing radiator panel, or facesheet. This paper focuses primarily on the thermal analysis considerations; the mechanical design is discussed separately. ${ }^{14}$ Figure 1 shows a morphing radiator design which combines the transformation response of shape memory alloys with a thermally conductive and linearly elastic biasing structure, creating a radiator panel that reconfigures passively in response to changes in temperature. ${ }^{12}$ The radiator consists of a circular composite panel fixed along the panel line of symmetry at the root. Here a flow tube is attached, which conducts heat from the TCS working fluid into the radiator. A high-emissivity coating is applied on the inner (concave) surface, shown with dark shading, and a low-emissivity coating is applied on the outer (convex) surface, shown with light shading. SMAs attached to the outermost surface of the panel cause the radiator to morph between various shapes, altering its view factor to space. The panel temperature drives this process. When sufficiently cold, the radiator takes on the fully closed circular shape shown in Fig. 1a. As the radiator temperature increases due to a warmer ambient environment and/or increase in the heat load (from the fluid in the flow tube), the SMAs contract, which opens the radiator to a wider configuration as shown in Fig. 1b; the maximum heat rejection shape is depicted in Figure 1c. This morphing behavior is intended to be fully reversible. Together, the variable view factor and selective surface emissivity increase the turndown ratio of the radiator, as will be shown.

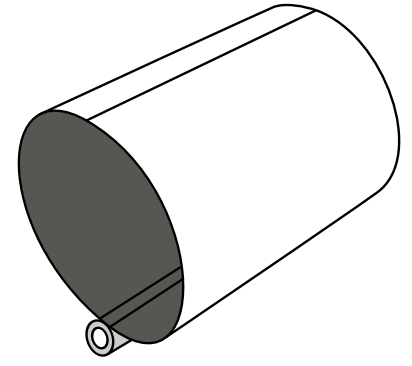

(a) Closed shape for minimum heat rejection.

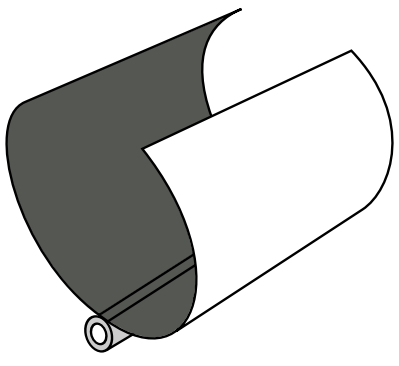

(b) Semi-open shape for intermediate heat rejection.

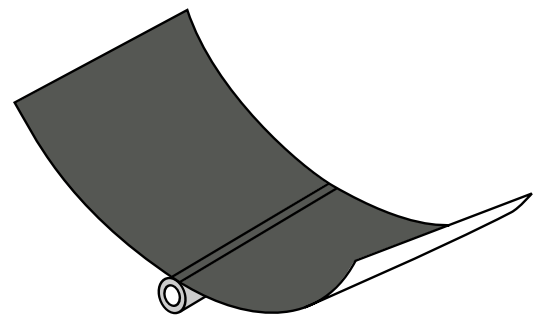

(c) Open shape for maximum heat rejection.

Figure 1: Schematic representation of a flexible morphing radiator panel. Light and dark shading represents low- and high-emissivity coatings, respectively. ${ }^{14}$

An array of morphing radiator panels is shown in Fig. 2, illustrating their application in a parallel-flow configuration. Hot fluid enters the radiator manifold via the inlet header, which distributes the working fluid among multiple parallel flow tubes. A series of individual morphing radiator panels are attached along each flow tube. The working fluid temperature decreases along the length of each tube as heat is rejected via radiation. In a warm environment (e.g. LEO), the working fluid and panel temperatures remain above the martensite start temperature, such that the SMAs are fully contracted and all panels are fully open. Thus, heat is rejected through the greatest possible radiator area via the high emissivity surface. In a cooler environment, the temperature near the downstream end of the flow tube decreases below the martensite transformation temperature, causing the SMAs to expand. This allows the panels to close, minimizing heat rejection. As a result of such behavior, this radiator design will tend to maintain a minimum fluid temperature in the range of the transformation temperatures of the SMA (i.e., between the austenite and martensite finish temperatures ${ }^{15}$ ), which can vastly simplify the overall design of the TCS. 


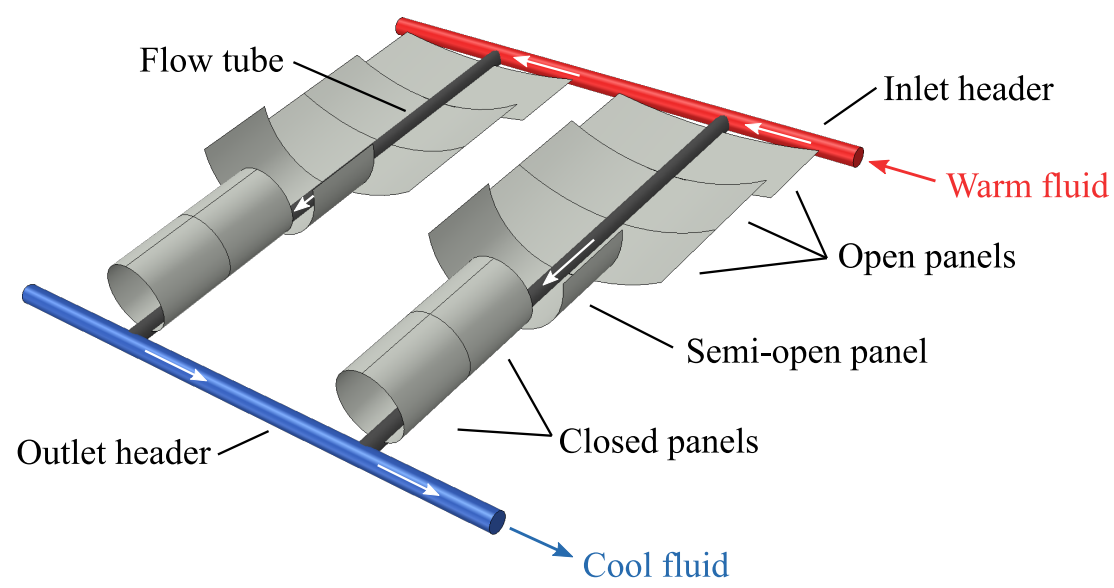

Figure 2: Illustration of an array of radiator panels in a parallel flow configuration. Arrows indicate fluid flow direction.

This concept featured a number of design challenges. In particular the face sheet required three contradictory material characteristics: adequate thermal conductivity to transport heat out of the fluid loop, ample flexibility to deform to its open hot shape, and sufficient stiffness to provide spring force for returning the radiator to its closed cold shape. Figure 3 shows the prototype morphing radiator used in the experimental studies in $2015 .{ }^{16}$ This prototype was designed to use wire-type SMAs to achieve the temperature-induced morphing behavior depicted above. (Note that Fig. 3a corresponds to Fig. 1c, with the SMAs in their austenitic (high-temperature) state.) The primary component in this prototype is a compliant and thermally conductive copper panel ( 7 in long, 3 in wide, 0.007 in thick), which was rolled along its length to form the semicircular shape shown in Fig. 3. Ten shape memory alloy wires rest against the outer surface of the panel and are fixed at each end to the straight edges of the panel with a pair of terminal blocks fabricated from 0.25 in square aluminum stock. The wires are otherwise unconstrained, allowing them to slide along the panel as they transform locally. Benchtop tests indicated the need for an additional biasing force beyond that provided by the copper to drive the panel towards the closed circular shape under cooling. A 1 in wide, 0.007 in thick 1095 steel closing spring was attached to the convex side of the panel for this purpose. In order to increase the rate of heat rejection via radiation in the open shape, the inside surface of the copper panel was painted with Aeroglaze $\mathrm{Z}^{3} 07^{\circledR}$, a flexible, high-emissivity polyurethane coating. The outside of the panel remained unpainted. The emissivities of the Aeroglaze Z307 paint, unpainted copper, and unpainted steel were measured to be $0.943,0.047$, and 0.143 respectively, with a Surface Optics Corp. ET- $100^{\circledR}$ emissometer. The copper panel was attached to a 0.375 in diameter stainless steel flow tube using a thermally conductive epoxy which allowed the radiator to be integrated into a pumped fluid loop for the experiment. The prototype was tested in a thermal vacuum environment where it demonstrated the morphing behavior under a range of heat loads and achieved a turndown ratio of 6.4:1 ${ }^{16}$

The goal of the efforts described here (and in the accompanying work ${ }^{14}$ ) was to improve beyond the preliminary prototype and study of 2015, producing a new device comprised of more advanced composite materials and SMA components with transition temperatures better tuned to the thermal vacuum environment. A carbon-fiber facesheet was developed for this purpose. Studies were done to optimize the design, taking into consideration fiber orientation and SMA integration. Prototype facesheets were fabricated using multiple plies of CSW T40-800/5320-1, a unidirectional prepreg tape designed for out-of-autoclave curing. ${ }^{17}$ The full process of material selection, manufacturing, and fatigue testing of these advanced morphing panels is described in the accompanying paper. ${ }^{14}$ The following sections describe a benchtop demonstration and thermal vacuum testing of these prototypes. 


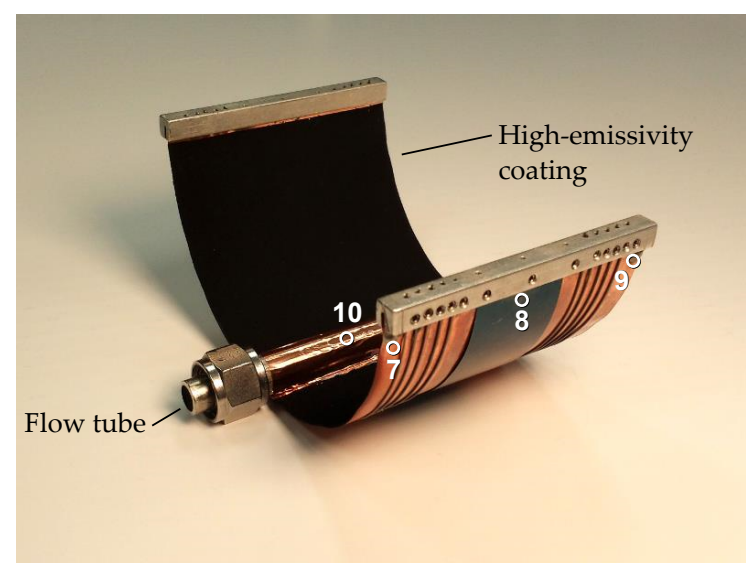

(a) Outside of prototype showing primary components.

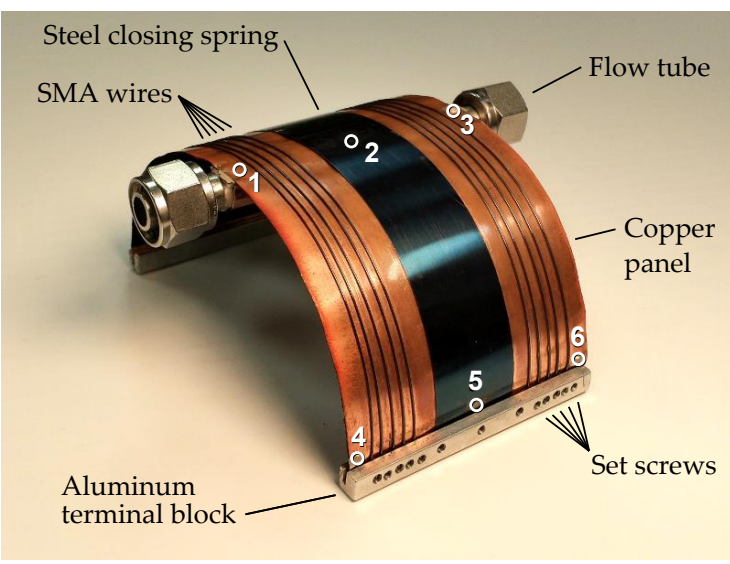

(b) Inside of prototype showing high-emissivity polyurethane coating.

Figure 3: Prototype morphing radiator test article from previous 2015 studies ${ }^{16}$ Panel is at room temperature demonstrating open shape. Open circles show locations where thermocouples were attached for testing.

\section{Benchtop Prototype}

After fabrication and prior to thermal vacuum testing, a benchtop prototype was fabricated as a proof-of-concept. This prototype (shown in Fig. 4) featured a number of SMA wires in the martensite phase at room temperature (i.e. the panel was in the closed configuration at room temperature (Fig. 4a)). The panel was heated using two heat guns to simulate the heat load from the fluid loop on the spacecraft. The increase in temperature caused the phase transformation in the SMA wires, driving the panel to its open configuration (Fig. 4b). When the heat was removed the composite cooled convectively in the laboratory environment and returned to the initial closed configuration. In this manner, the shape of the radiator was controlled by thermal loading exclusively, and the morphing behavior was demonstrated in an ambient room temperature setting.

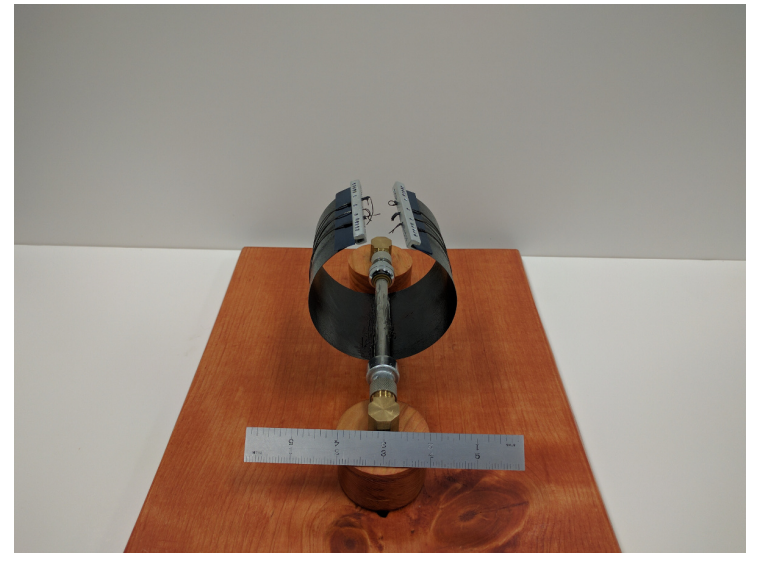

(a) Closed configuration corresponding to minimum temperature.

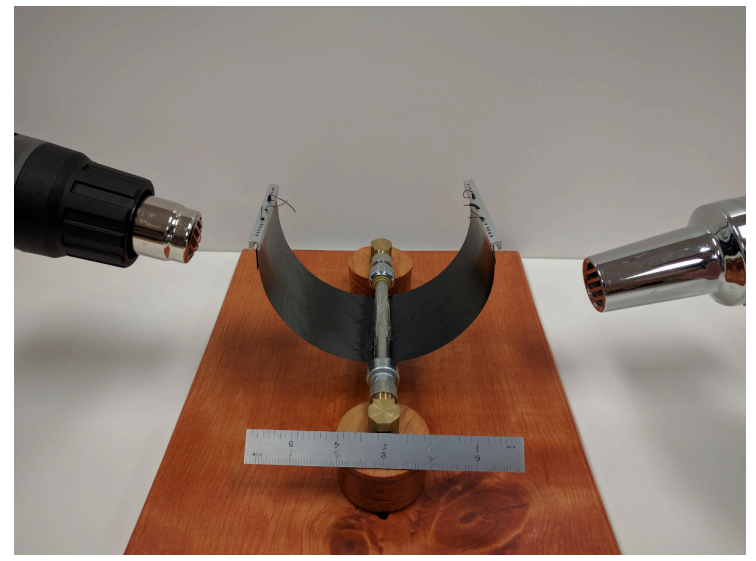

(b) Open configuration corresponding to maximum temperature, resulting from heat provided by two heat guns.

Figure 4: A demonstration of the benchtop prototype. 


\section{Thermal Vacuum Chamber Tests}

\section{A. Test Articles}

Following the benchtop proof-of-concept, three different test articles were fabricated for testing in a thermal vacuum environment. Table 1 gives an overview of the characteristics of each test article. The first row describes the carbon fiber ply layering (i.e. the layup) as a sequence of ply orientation angles within each composite panel. The ply orientation angles were defined with respect to the circumferential direction (i.e. the fibers of a $0^{\circ}$ ply are along the circumference of the panel and the fibers of a $90^{\circ}$ ply are oriented parallel to the flow tube). The key mechanical and thermal properties of the panel, including bending stiffness and effective conduction coefficient per unit length (denoted by $K_{t}$ ), are also listed for each panel, along with the primary dimensions. More details of these aspects of panel design are provided in the accompanying work.$^{14}$

Table 1: Overview of the three test articles fabricated for the thermal vacuum chamber tests.

\begin{tabular}{rlll}
\hline & Test Article A & Test Article B & Test Article C \\
\hline Layup & {$[90 / 45 / 0 / 45 / 90]$} & {$[90 / 45 / 0 / 0 / 45 / 90]$} & {$[90 / 45 / 0 / 0 / 0 / 45 / 90]$} \\
Bending stiffness & $0.44 \mathrm{~Pa} \cdot \mathrm{m}^{3}$ & $0.82 \mathrm{~Pa} \cdot \mathrm{m}^{3}$ & $1.42 \mathrm{~Pa} \cdot \mathrm{m}^{3}$ \\
Cond. coeff. per unit width $\left(K_{t}\right)$ & $0.091 \mathrm{~W} / \mathrm{K}$ & $0.121 \mathrm{~W} / \mathrm{K}$ & $0.152 \mathrm{~W} / \mathrm{K}$ \\
Closed diameter & $3.0 \mathrm{in}$ & $3.0 \mathrm{in}$ & $3.5 \mathrm{in}$ \\
Width & $3.0 \mathrm{in}$ & $3.0 \mathrm{in}$ & $4.0 \mathrm{in}$ \\
SMA type \& qty. & Wires $(\mathrm{x} 18)$ & Wires $(\mathrm{x} 36)$ & Strips $(\mathrm{x} 8)$ \\
SMA phase at room temp. & Austenite & Austenite & Martensite \\
\hline
\end{tabular}

The first two test articles, A and B, were nearly identical apart from the layup. Test Article A incorporated a layup with a single $0^{\circ}$ ply, while the layup for Test Article B included two $0^{\circ}$ plies. Both of these test articles utilized SMA wires in the austenite (high-temperature) phase at room temperature, requiring Test Articles A and B to be assembled in their open configurations. For Test Article A, a single SMA wire was threaded through each hole in the terminal blocks for a total of 18 wires. Since Test Article B featured two $0^{\circ}$ plies the bending stiffness was approximately twice that of Article A (see Table 1), thus requiring approximately twice the force to open to the same radius. To maintain a comparable level of stress in the wires between the two test articles, two SMA wires were threaded through each hole in the terminal block on Test Article B. Figure 5 shows Test Article A prior to thermal vacuum testing.

After attaching the wires to the panels, the flow tubes were attached using thermal adhesive and secured with a thermally insulated tape. Thermocouples were attached to the facesheet at various locations to record temperature across the panel.

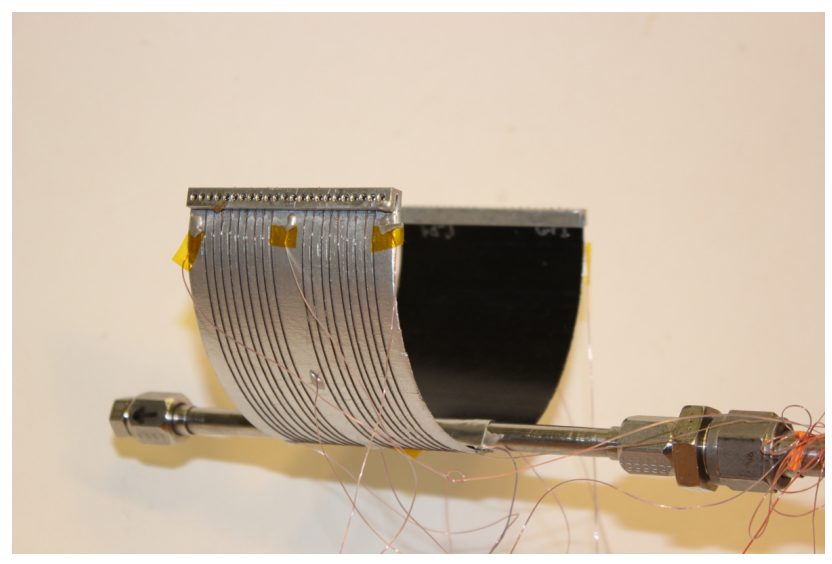

Figure 5: Test Article A featuring SMA wires installed in a stressed (open) configuration.

Test Article C was slightly larger than Test Articles A and B and used SMA strips instead of wires. Whereas the SMA wires were in the austenite phase at room temperature, the SMA strips used in Test Article C were in the martensite (low-temperature) phase at room temperature. Thus, Article $\mathrm{C}$ was assembled in the closed configuration. Prior to assembly, each strip was detwinned at $300 \mathrm{MPa}$ to generate the transformation strain needed for austenite shape 
recovery during heating. This process involved submerging the strips in liquid nitrogen to reach the martensite phase throughout the material, and then applying 300MPa of axial tension to each strip. Figure 6 shows Test Article C fully assembled.
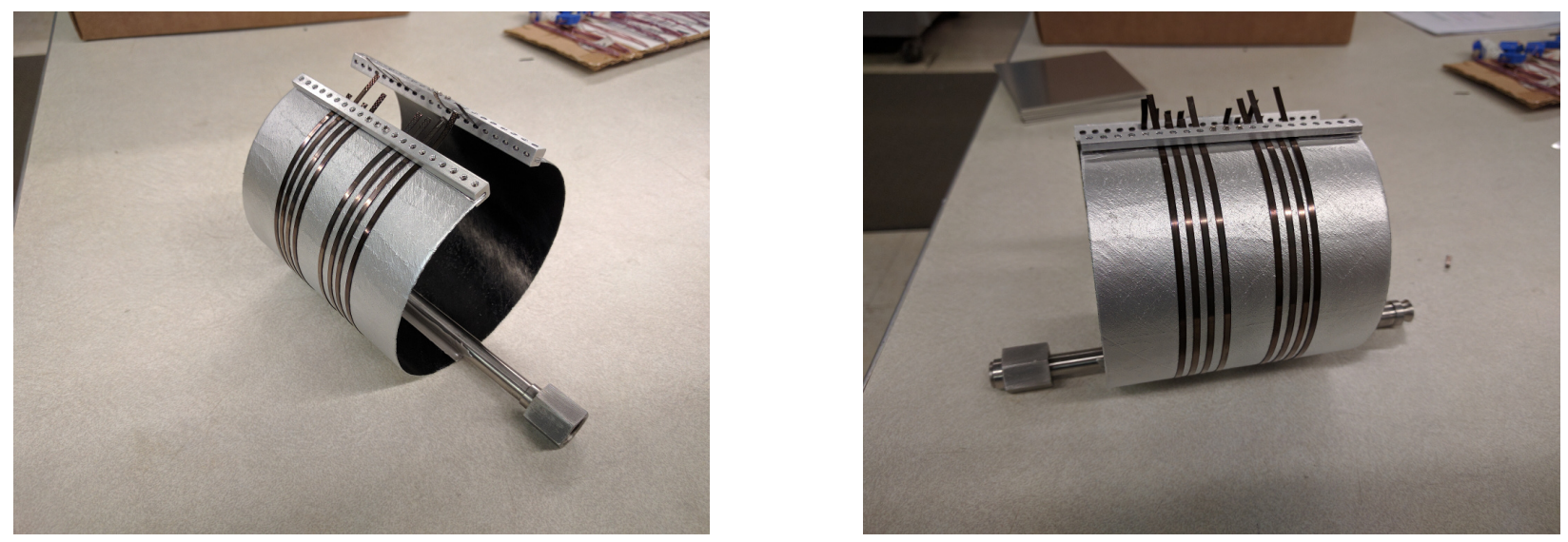

Figure 6: Test Article C featuring SMA strips installed in a detwinned and stress free (closed) configuration.

\section{B. Test Setup}

The vacuum chamber used in the experiments was a small, high-vacuum thermal environment chamber located at NASA Johnson Space Center. Figure 7 shows a schematic diagram of the flow loop used in the thermal vacuum chamber tests. The primary component was an SP Scientific RC211 ${ }^{\circledR}$, a recirculating chiller shown on the left side of Figure 7. The chiller contains a pump and integrated heater and chiller modules, allowing the temperature of the working fluid to be controlled between $-45^{\circ} \mathrm{C}$ and $100^{\circ} \mathrm{C}$. The fluid loop used Dynalene $\mathrm{HC}-50^{\circledR}$ - a nontoxic water-based coolant with a freezing point below $-50^{\circ} \mathrm{C}$ - as the working fluid. The temperature bounds for the test were selected to ensure the fluid did not freeze during testing.

Beginning at the chiller, the fluid first passed through two parallel flow lines, one with an inline heater, and the other that bypassed the heater. These lines were opened and closed via ball valves during the different phases of experiment to adjust the flow temperature as desired. Downstream of these valves the fluid passed through a Coriolis flow meter manufactured by Micro Motion ${ }^{\circledR}$, which was used to measure the instantaneous flow rate throughout the experiments. The fluid then entered the vacuum chamber and passed through the flow tube contacting the test article. Two immersion thermocouples, shown with numbered circles in Fig. 7, were used to measure the fluid temperatures at the inlet and outlet of the radiator flow tube. Finally, the fluid exited the vacuum chamber, passed through a check valve, and returned to the chiller.

Figure 8 shows the thermal vacuum chamber, chiller cart, and fluid loop. Figure 9 shows a close-up of the test section inside the chamber with the radiator installed into the fluid loop.

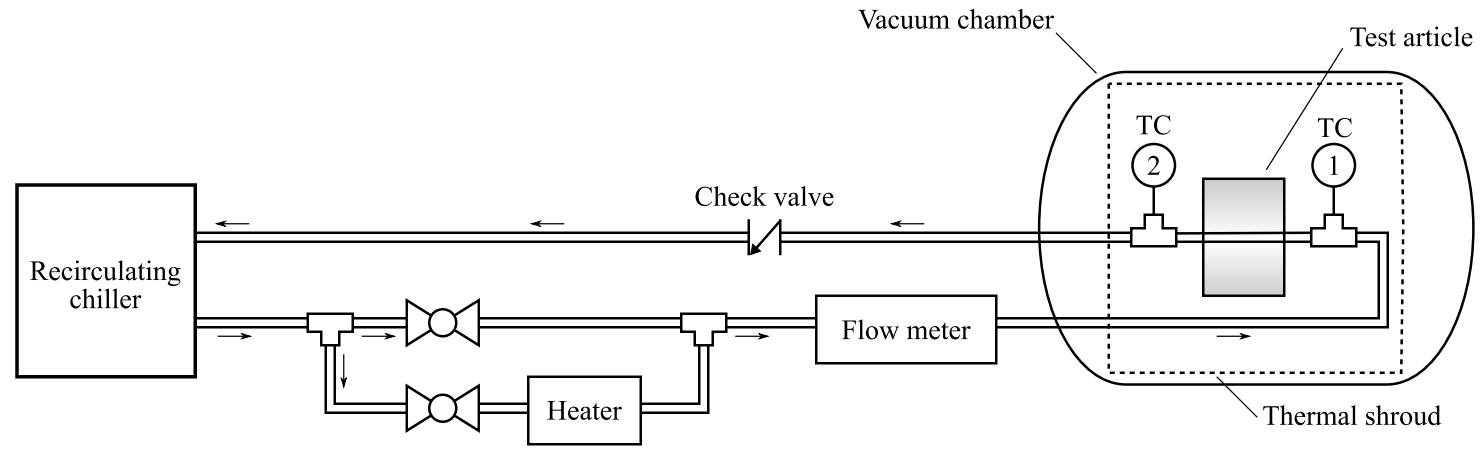

Figure 7: Schematic diagram showing experimental setup for the thermal vacuum chamber test. 


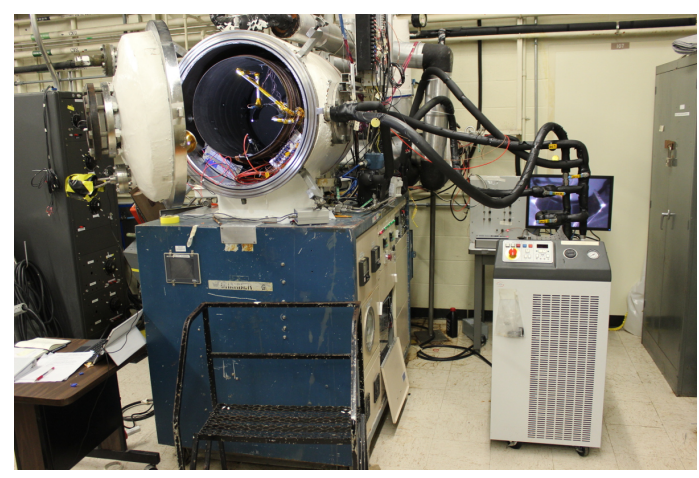

(a) Test setup showing thermal vacuum chamber, chiller cart, and exterior fluid lines.

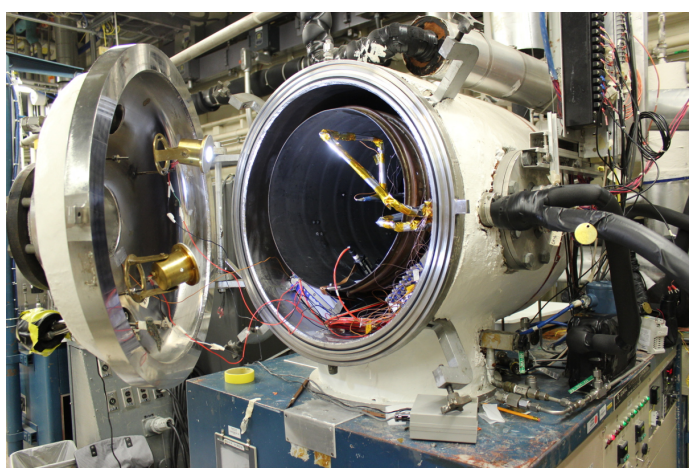

(b) Closer view of thermal vacuum chamber test section showing interior fluid lines and LED lamps.

Figure 8: Photographs of the vacuum chamber test setup.

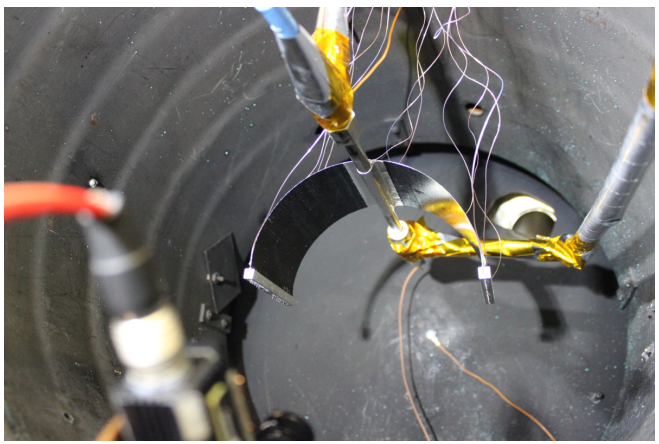

Figure 9: Close-up of morphing radiator test article after being installed in the fluid loop (Test Article B shown).

\section{Test Procedure}

Prior to the start of each test, the appropriate test article was mounted to the flow line inside the chamber (see Fig. 7). The chamber was then sealed, a vacuum established, and the fluid lines opened. The fluid temperature was cycled over a $\sim 3$ hour period, during which time the morphing behavior was observed. Temperature data was recorded at various locations on the panel and flow tube by the thermocouples throughout the test. Additionally, a camera mounted outside a window on the vacuum chamber captured images of the panel at 2 second intervals throughout the heat cycling process. Figures 10 and 11 show Test Article A inside the thermal vacuum chamber during testing.

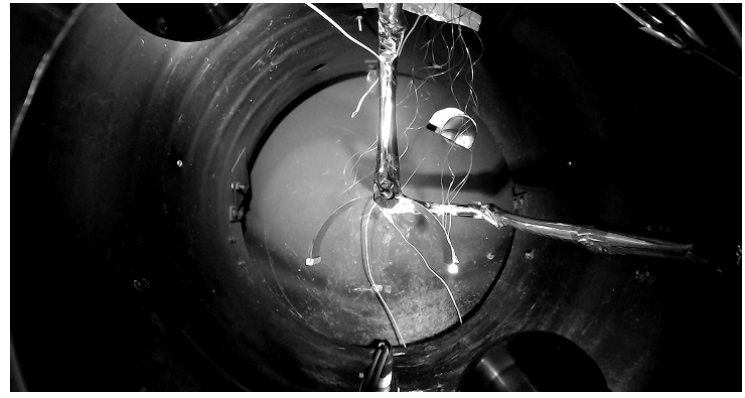

(a) Open configuration corresponding to maximum fluid temperature.

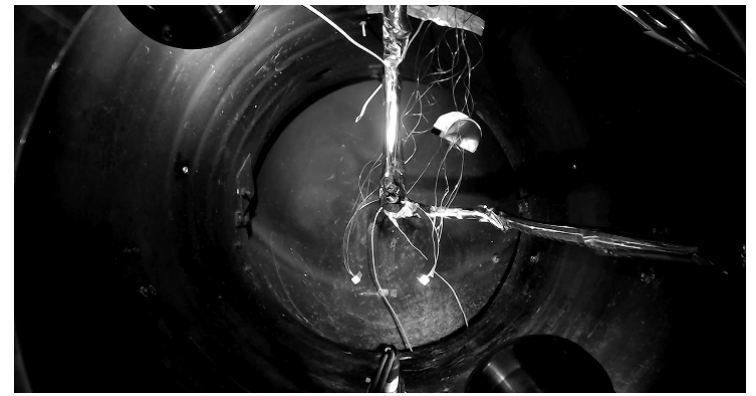

(b) Closed configuration corresponding to minimum fluid temperature.

Figure 10: Images of Test Article A in the vacuum chamber photographed by the camera outside the chamber. 


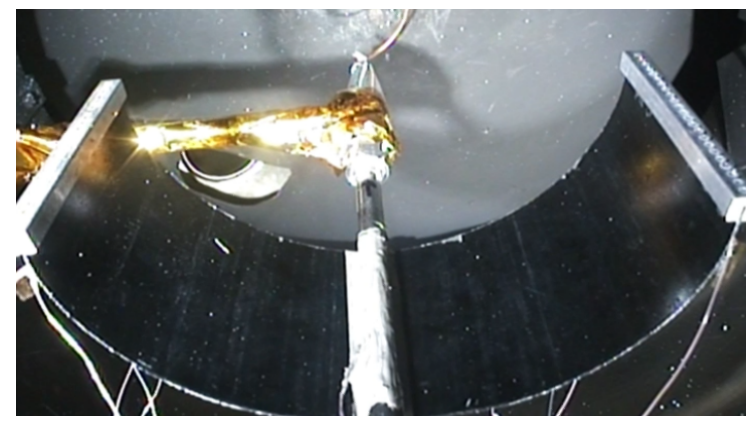

(a) Open configuration corresponding to maximum fluid temperature.

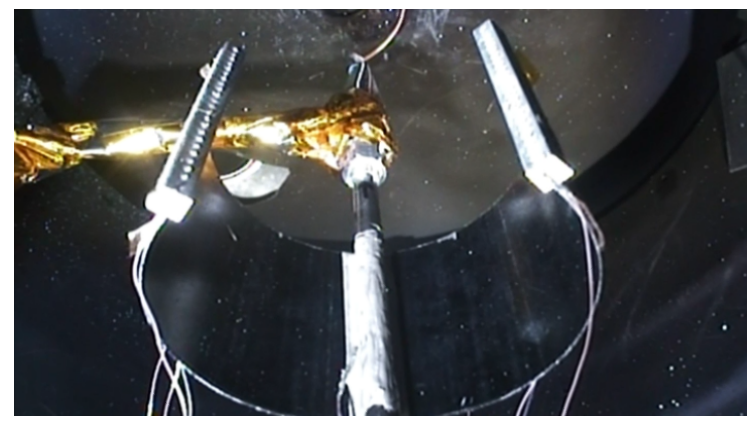

(b) Closed configuration corresponding to minimum fluid temperature.

Figure 11: Images of Test Article A in the vacuum chamber captured by the in-chamber camera.

\section{Analysis of Experimental Data}

A custom MATLAB ${ }^{\circledR}$ script processed the images of the test articles captured during the test, computing the panel radius over time via a least squares regression technique. Test Articles A and B achieved a range in panel radius of 1 inch and 1.1 inches respectively. Test Article $\mathrm{C}$ only achieved a radius range of 0.4 inches.

As the radius increased with fluid temperature, the view factor of the internal panel surface increased, thereby increasing the total radiative heat rejection of the panel. Thus, the range of radii directly drove the range of achievable heat rejection rates, establishing a turndown ratio for the panel. To this end, the analysis of heat rejection centered around Test Article B, which achieved the widest range in radius. The temperature histories and panel radius over time are shown for Test Article B in Figure 12.

A thermal finite element model of Test Article B was developed in ABAQUS ${ }^{\circledR}$. By specifying the panel radius and boundary conditions known at various instances in the thermal vacuum test, a thermal analysis was performed which provided the steady-state temperature distribution and heat rejection rate of Article B at each instance. From these simulations the maximum and minimum heat rejections of Test Article B were determined; thus allowing calculation of the turndown ratio. The simulation also directly calculated the minimum temperature at the free edges of the panel (i.e. at the terminal blocks) to compare with the minimum panel temperature recorded in the thermal chamber test.

The time history of heat rejection throughout the testing process for Test Article B is shown in Fig. 13a. The comparison of minimum panel temperature between these predictions and the actual data from Test 2 is shown in Fig. 13b. The overall accuracy of the minimum temperature predictions serves as a meaningful validation of the thermal model and the related results that follow. In the open configuration (hot phase, maximum radius) the model calculated a heat rejection rate of $9.97 \mathrm{~W}$, with a discrepancy in minimum temperature results of only $\sim 7^{\circ} \mathrm{C}$. In the closed configuration (cold phase, minimum radius) a heat rejection rate of $2.06 \mathrm{~W}$ was calculated, with a temperature discrepancy of $\sim 3^{\circ} \mathrm{C}$. Thus, the overall turndown ratio was calculated at 4.84:1. The larger temperature discrepancy for the hot phase was likely due to the large temperature gradient existing between the radiator root (point of contact with the flow tube) and the terminal blocks $\left(\sim 26^{\circ} \mathrm{C}\right)$. This gradient is much less significant in the cold phase, with a temperature variation of $\sim 4^{\circ} \mathrm{C}$, explaining the smaller temperature discrepancy in the cold phase.

As a comparison, the turndown ratio was computed for Article B without including changes due to morphing. The radiator model was given the same radius and sink temperature for which the maximum heat rejection was computed in Fig. 13a, while the root temperature (the panel temperature boundary condition) was that of the minimum heat rejection case. From this simulation the turndown ratio without morphing was found to be 4.32:1. This demonstrated an increase in turndown capacity through the morphing behavior.

Earlier in this paper it was stated that the highest turndown ratio of a state-of-the-art TCS was 3:1. It is important to reiterate that this is relative to the particular thermal environment in which said thermal control system operates (indicated above to be Low Earth Orbit). The turndown ratios achieved by the morphing radiator are particular to the thermal environments experienced in the thermal vacuum chamber. Though similar, the environments of the two systems are not identical, which should be noted when comparing their turndown ratios.

Regarding the turndown ratio achieved by the morphing radiator, 4.84 is lower than required for an actual production system (targeted between 6:1 and 12:1 as mentioned earlier). This turndown ratio was largely impacted by heat that escaped through the circular open ends of the radiator during the testing process; a source of uncontrolled heat rejection. Previous design studies have shown that the addition of low-emissivity side caps would block heat rejection through the 
open ends and significantly reduce heat losses, and that long cylindrical radiators with side caps are in fact needed to ensure optimal performance.$^{18}$ Future work will involve modeling the radiator with these features to explore the effects on turndown capacity.

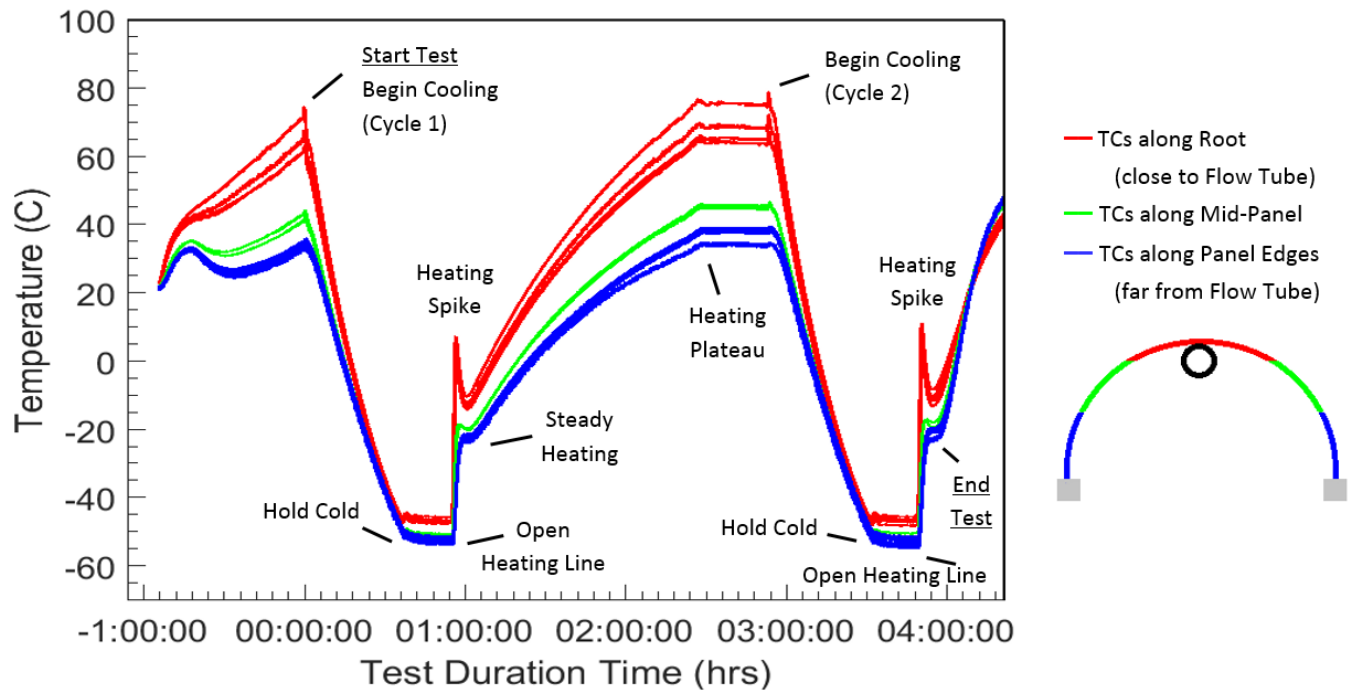

(a) Time history of Test Article B panel temperature during testing. Thermocouples were attached to the radiator at various locations, resulting in the range of temperatures shown.

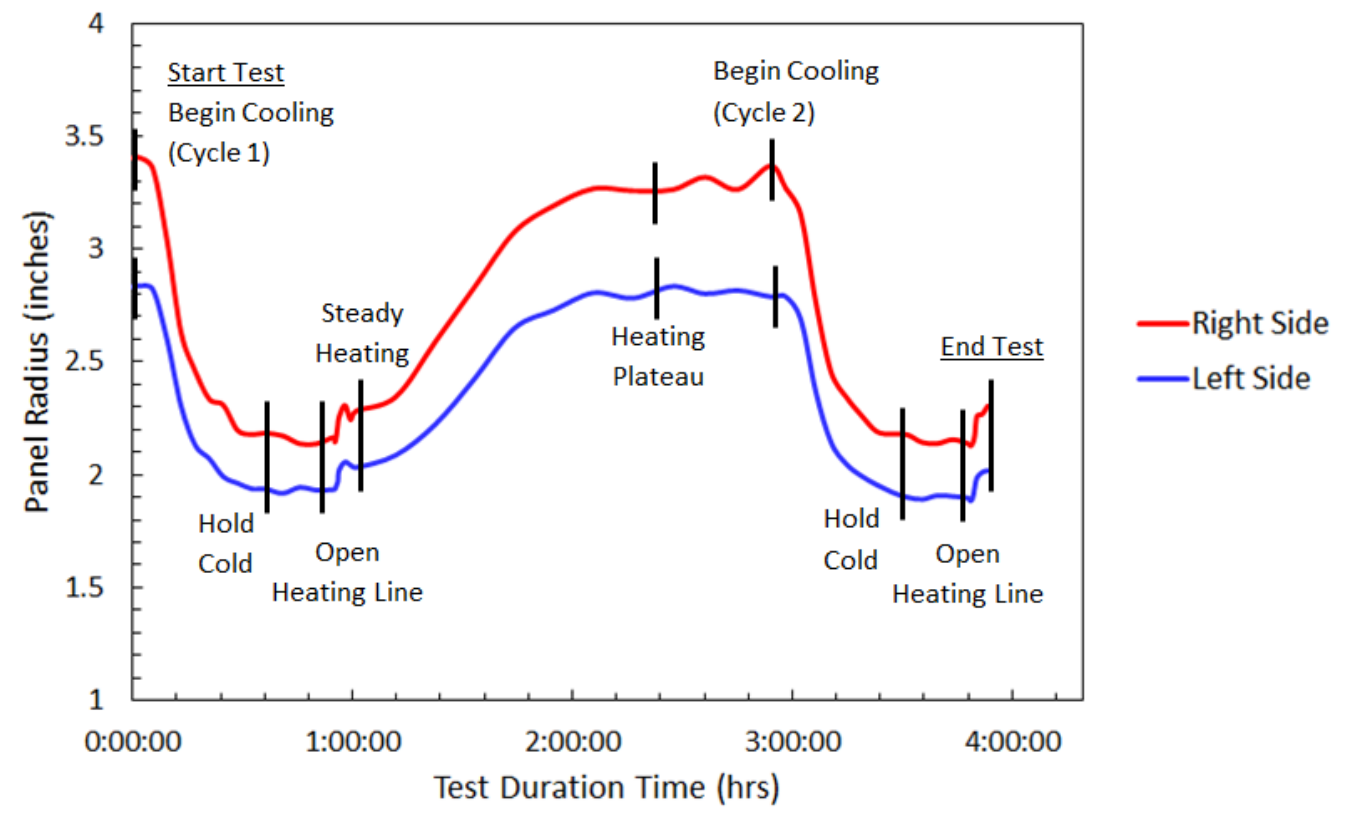

(b) Time history of Test Article B panel radius during testing. The radius of curvature of each side of the panel is shown.

Figure 12: Experimental results from the test of Article B (the prototype with the strongest morphing response). 


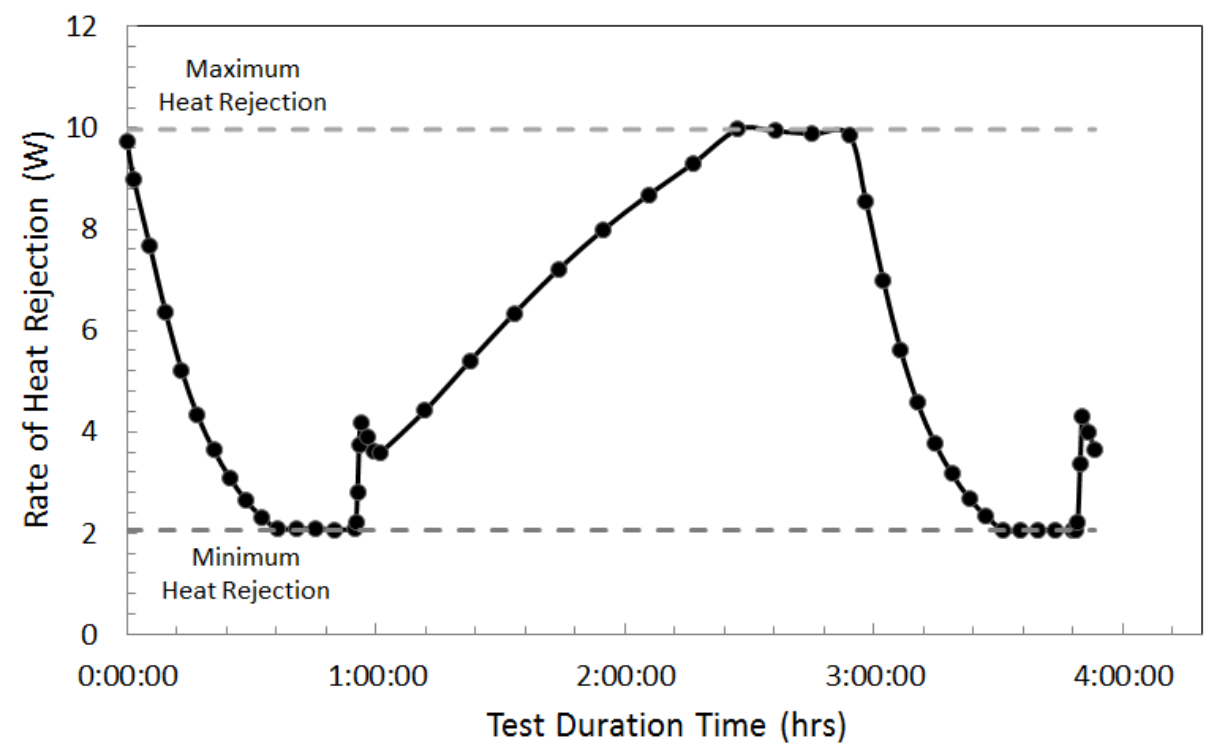

(a) Rate of heat rejection of Test Article B throughout thermal vacuum test.

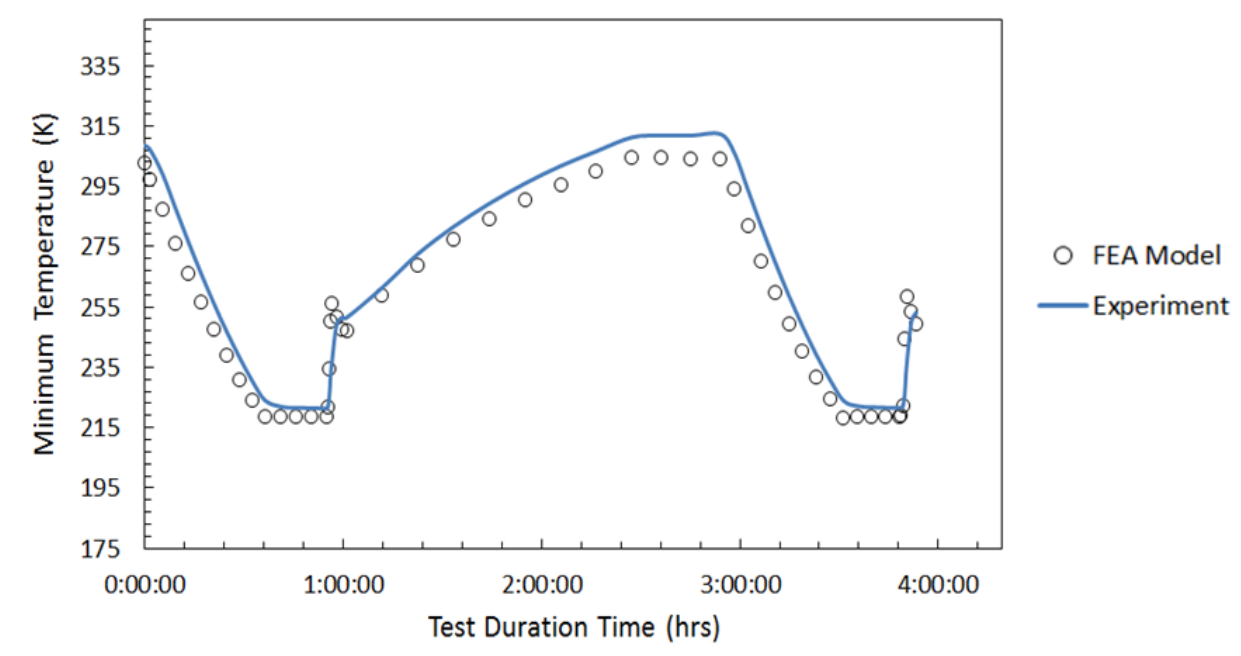

(b) Comparison of minimum panel temperature calculated in Abaqus simulation with that measured in the thermal vacuum experiment.

Figure 13: Results from thermal analysis of Test Article B model.

\section{Summary \& Future Work}

With the increasing need for advanced thermal control systems a variable heat rejection radiator offers a unique solution. A composite morphing radiator actuated with shape memory alloys was designed to achieve this purpose. Multiple prototypes were developed and tested in a thermal vacuum environment where they exhibited the desired temperature-induced actuation behavior. Test Article B, featuring two circumferentially oriented carbon fiber plies and 36 SMA wires achieved the widest range in radius during the testing process and offered a turndown ratio of 4.84:1. The morphing behavior was shown to aid the turndown capacity, which was 4.32:1 without morphing.

These efforts have shown that SMA materials are capable of morphing a composite radiator panel through a range of shape configurations, allowing for variable heat rejection above that achieved by modern thermal control systems. Moving forward, the project will focus on design improvements involving SMA and flow tube integration to improve heat transfer between the working fluid and the panel, thus improving the morphing response. Furthermore, the radiator will be modeled with side caps to reduce uncontrolled heat rejection and increase the overall turndown capacity of the 
design. These efforts will not only contribute to the developments of new adaptive space structures, but will further advance the next generation of thermal control systems, supporting continued space exploration close to home and beyond.

\section{Acknowledgments}

Special thanks to the following: Othmane Benefan (NASA Glenn) for providing the SMA wires and strip; James Brown, Tobin Barnes, and Peter Grenfell (NASA JSC, Innovation Design Center) for fabricating the terminal blocks for the radiator prototypes; Joe Settles (NASA JSC) for assistance in detwinning the SMA strips prior to assembling Test Article C; and the numerous individuals at NASA JSC who helped with setting up and running the vacuum chamber experiments in Chamber G. Additional thanks to Matthew Wescott, Brent Bielefeldt, and Madalyn Mikkelsen for their contributions to this paper.

\section{References}

1 "Appendix C: Technology Development for Variable Heat Rejection," Game Changing Opportunities in Technology Development, NASA, 2012, NASA Research Announcement NNL12A3001N.

2 Stephan, R. A., “Overview of NASA's thermal control system development for exploration project,” SAE ICES, 2009.

3 Ochoa, D. A., Vonau, W., and Ewert, M. K., "A Comparison between One- and Two-Loop ATCS Architectures Proposed for CEV," Tech. rep., SAE Technical Paper, 2009.

${ }^{4}$ NASA, “TA 14: Thermal Management Systems,” NASA Technology Roadmaps, July 2015.

${ }^{5}$ Hill, S. A., Kostyk, C., Motil, B., Notardonato, W., Rickman, S., and Swanson, T., “Thermal Management Systems Roadmap: Technology Area 14,” Tech. rep., NASA, July 2015.

6 Juhasz, A. J. and Peterson, G. P., "Review of advanced radiator technologies for spacecraft power systems and space thermal control," NASA STI/Recon Technical Report N, Vol. 94, 1994.

${ }^{7}$ Ganapathi, G. B., Sunada, E. T., Birur, G. C., Miller, J. R., and Stephan, R., "Design Description and Initial Characterization Testing of an Active Heat Rejection Radiator with Digital Turn-Down Capability," Tech. rep., SAE Technical Paper, 2009.

${ }^{8}$ Bannon, E. T., Bower, C. E., Sheth, R., Stephan, R., Chandrasekhar, P., and Zay, B., "Electrochromic radiator coupon level testing and full scale thermal math modeling for use on altair lunar lander," 40th International Conference on Environmental Systems, 2010, p. 6110.

${ }^{9}$ Demiryont, H. and Moorehead, D., "Electrochromic emissivity modulator for spacecraft thermal management," Solar Energy Materials and Solar Cells, Vol. 93, No. 12, 2009, pp. 2075-2078.

${ }^{10}$ Metts, J. G., Nabity, J. A., and Klaus, D. M., "Theoretical performance analysis of electrochromic radiators for space suit thermal control," Advances in Space Research, Vol. 47, No. 7, 2011, pp. 1256-1264.

${ }^{11}$ Benkahoul, M., Chaker, M., Margot, J., Haddad, E., Kruzelecky, R., Wong, B., Jamroz, W., and Poinas, P., “Thermochromic VO 2 film deposited on Al with tunable thermal emissivity for space applications," Solar Energy Materials and Solar Cells, Vol. 95, No. 12, 2011, pp. 3504-3508.

${ }^{12}$ Cognata, T., Hartl, D., Sheth, R., and Dinsmore, C., "A Morphing Radiator for High-Turndown Thermal Control of Crewed Space Exploration Vehicles," Proceedings of 23rd AIAA/AHS Adaptive Structures Conference, Kissimmee, FL, 2015.

${ }^{13}$ Lagoudas, D., editor, Shape Memory Alloys: Modeling and Engineering Applications, Springer-Verlag, New York, 2008.

${ }^{14}$ Wescott, M. T., McQuien, J. S., Bertagne, C. L., Whitcomb, J. D., Hartl, D. J., and Erickson, L. R., "Design and Fabrication of a Composite Morphing Radiator Panel Using High Conductivity Fibers," Proceedings of 25th AIAA/AHS Adaptive Structures Conference, Grapevine, TX, 2017.

${ }^{15}$ Lagoudas, D., Hartl, D., Chemisky, Y., Machado, L., and Popov, P., “Constitutive Model for the Numerical Analysis of Phase Transformation in Polycrystalline Shape Memory Alloys,” International Journal of Plasticity, Vol. 32-33, 2012, pp. $155-183$.

${ }^{16}$ Bertagne, C. L., Chong, J. B., Whitcomb, J. D., Hartl, D. J., Erickson, L. R., and Sheth, R. B., "Experimental Characterization of a Shape Memory Alloy-Based Morphing Radiator," Proceedings of 24th AIAA/AHS Adaptive Structures Conference, San Diego, CA, 4-8 January 2016, p. 1568.

${ }^{17}$ Kellas, S., Lerch, B. A., and Wilmoth, N., "Mechanical Characterization of In and Out-of-Autoclave Cured Composite Panels for Large Launch Vehicles," 2012.

${ }^{18}$ Bertagne, C. L., Hartl, D. J., Cognata, T. J., Sheth, R. B., and Dinsmore, C. E., "Testing and Analysis of a Morphing Radiator Concept for Thermal Control of Crewed Space Vehicles," 2015. 